\title{
Exercise physiology in left ventricular assist device patients: insights from hemodynamic simulations
}

\author{
Libera Fresiello $^{1,2}$, Christoph Gross ${ }^{3}$, Steven Jacobs $^{1}$ \\ ${ }^{1}$ Department of Cardiovascular Sciences, Cardiac Surgery, Katholieke Universiteit Leuven, Leuven, Belgium; ${ }^{2}$ Institute of Clinical Physiology, \\ National Research Council, Pisa, Italy; ${ }^{3}$ Center for Medical Physics and Biomedical Engineering, Medical University of Vienna, Vienna, Austria \\ Correspondence to: Libera Fresiello. Cardiale Heelkunde, UZ Herestraat 49 - bus 7003, 3000 Leuven, Belgium. Email: libera.fresiello@gmail.com.
}

Left ventricular assist devices (LVADs) assure longer survival to patients, but exercise capacity is limited compared to normal values. Overall, LVAD patients show high wedge pressure and low cardiac output during maximal exercise, a phenomenon hinting at the need for increased LVAD support. Clinical studies investigating the hemodynamic benefits of an LVAD speed increase during exercise, ended in inhomogeneous and sometimes contradictory results. The native ventricle-LVAD interaction changes between rest and exercise, and this evolution is complex, multifactorial and patient-specific. The aim of this paper is to provide a comprehensive overview on the patient-LVAD interaction during exercise and to delineate possible therapeutic strategies for the future. A computational cardiorespiratory model was used to simulate the hemodynamics of peak bicycle exercise in LVAD patients. The simulator included the main cardiovascular and respiratory impairments commonly observed in LVAD patients, so as to represent an average hemodynamic response to exercise. In addition, other exercise responses were simulated, by tuning the chronotropic, inotropic and vascular functions, and implementing aortic regurgitation and stenosis in the simulator. These profiles were tested under different LVAD speeds and LVAD pressure-flow characteristics. Simulations output showed consistency with clinical data from the literature. The simulator allowed the working condition of the assisted ventricle at exercise to be investigated, clarifying the reasons behind the high wedge pressure and poor cardiac output observed in the clinics. Patients with poorer inotropic, chronotropic and vascular functions, are likely to benefit more from an LVAD speed increase during exercise. Similarly, for these patients, a flatter LVAD pressure-flow characteristic can assure better hemodynamic support under physical exertion. Overall, the study evidenced the need for a patient-specific approach on supporting exercise hemodynamics. In this frame, a complex simulator can constitute a valuable tool to define and test personalized speed control algorithms and strategies.

Keywords: Exercise capacity; ventricular assist device; cardiovascular model

Submitted Oct 20, 2020. Accepted for publication Feb 01, 2021.

doi: 10.21037/acs-2020-cfmcs-23

View this article at: http://dx.doi.org/10.21037/acs-2020-cfmcs-23

\section{Introduction}

\section{Left ventricular assist device (LVAD) therapy}

Over the last two decades, the use of LVADs has increased tremendously. Over the last decade, continuous flow devices have evolved to become the most used ones, with more than $95 \%$ of the implanted devices now being this type $(1,2)$.

\section{Hemodynamics of LVAD patients}

The purpose of the LVAD is to support the cardiac output (CO) of heart failure (HF) patients. Although CO normalizes, the arterial pressure in LVAD patients is quite different from patients without an assist device. A continuous flow increases the mean arterial pressure, mainly due to the increase of diastolic blood pressure (3). 
Because there is an increased risk of stroke if the systolic pressure exceeds $100 \mathrm{mmHg}$, most LVAD centers will treat their patients with antihypertensive drugs to keep the systolic pressures below this value (4). Since the LVAD actively drains blood from the ventricle, the ventricle in LVAD patients is preload deprived. According to the Frank-Starling mechanism, this leads to a lesser pressure developed in systole (5). On top of that, the LVAD increases the arterial pressures, thus increasing the afterload for the ventricle. This can lead to a state in which the aortic valve doesn't open anymore, or only intermittently. LVAD therapy also decreases the pulmonary artery pressures and pulmonary capillary wedge pressure (PCWP) $(6,7)$. This is also the case in many patients with fixed pulmonary hypertension, giving them the opportunity to be transplanted after LVAD therapy (8).

\section{LVAD and organ function}

LVAD therapy improves end organ function to a certain extent in HF patients. Kidney function improves in the first months after implantation in most patients, only in a minor subset of patients these changes are present in the longterm follow-up (9). Liver function has been studied less in LVAD patients. Patients with preoperative impairment in liver function showed a recovery after LVAD implant (10).

Despite a survival benefit in HF patients (11), LVAD therapy is associated with complications, especially stroke, infection, bleeding and organ dysfunction (12). LVAD patients report an improved physical activity and quality of life but this improvement is still inferior to that of patients who received a heart transplant $(13,14)$. Third generation LVADs with magnetic levitation of the rotor are now showing comparable three-year survival rates to transplantation (15). The increasing use of LVADs as a destination therapy and the increased long-term survival will make reducing complications and increasing quality of life one of the major challenges in the future of LVAD development.

\section{Clinical observations during exercise}

Oxygen consumption at peak exercise $\left(\mathrm{pVO}_{2}\right)$, measured with cardiopulmonary exercise testing, is used for a wide spectrum of clinical applications, ranging from the assessment of athletes' performance to the evaluation of cardiorespiratory impairments (16). Cardiopulmonary exercise testing is highly recommended for treatment evaluation in advanced stage HF patients and useful for patients' risk assessment and stratification for risk of HF (17). The results of these tests, also referred to as maximal exercise capacity, encompass the entire body as a multi-organ system. Physiologic responses during cardiopulmonary exercise tests involve the somatic and autonomic nervous systems, imply an adequate functional response of the heart and the circulatory system, and require the lungs and the muscles to generate proper energy output according to the exercise intensity level.

The population of LVAD recipients contains advanced HF patients from a wide distribution of demographics and epidemiologic profiles on the continuous HF spectrum. Higher age and weight, deconditioning and HF with reduced ejection fraction are associated with lower $\mathrm{pVO}_{2}$, to name a few of the general impairments involved (17). Confounding factors for $\mathrm{pVO}_{2}$ are possibly implied in "LVAD implantation timing" or "HF time course" (18). Despite the success of normalizing hemodynamics at rest and improvements in survival after LVAD implantation, patients' $\mathrm{pVO}_{2}$ remain severely impaired at approximately $50 \%$ compared to age and gender matched controls (19). For this comparison it is noteworthy that most patients are too frail to even perform exercise capacity testing preLVAD. In patients able to perform exercise capacity testing prior to LVAD, one study reported significant increase in $\mathrm{pVO}_{2}$ after LVAD implantation whereas another reported no significant changes in $\mathrm{pVO}_{2}$ after implantation $(20,21)$.

\section{Maximal and submaximal exercise}

Submaximal exercise [measured through six-minute walk $(6 \mathrm{MW})]$ and functional capacity, were observed to improve in the first six months after LVAD implantation (22). Similarly, maximal exercise capacity, assessed by cardiopulmonary tests, improves in the first six months after LVAD implantation (23).

\section{Influence of LVAD speed on exercise capacity}

Exercise in LVAD patients was studied extensively for more than a decade $(19,24-26)$. One major focus of these studies was to analyse the influence of LVAD speed on exercise capacity, or $\mathrm{CO}$ at peak exercise as its surrogate. Therefore, various comparisons were performed: baseline speed $v s$. high speed, baseline speed $v s$. reduced speed, reduced speed $v s$. high speed and baseline speed constant $v s$. ramp speed increase $(19,24,25)$. Different results were reported and no 
clear benefit of LVAD speed on the complex interactions involved to improve exercise performance could be observed unanimously. Minor to no improvements with higher LVAD speeds compared to baseline were reported, whereas lower results were found with less cardiac support by lower LVAD speeds. Amongst the most important findings was the observation of a patient subgroup with a higher ejection fraction that showed less dependency on exercise output with LVAD speed reduction (27) as well as the finding that older LVAD patients showed more benefit in terms of increase in $\mathrm{pVO}_{2}$ with LVAD speed increase (28). Even though higher LVAD speeds at rest reduce pulmonary pressures, similar levels of PCWP for constant versus increased LVAD speed were reported (26). Nevertheless, improvements with increased LVAD speed compared to baseline speed were observed for the sub-maximal $6 \mathrm{MW}$ test. In summary the clinical observations showed less decrease in exercise performance with lower LVAD speeds for patients with a better left ventricular contractility, whereas in general no uniform trend of an increase in exercise performance with higher LVAD speeds compared to baseline speeds were observed.

\section{CO during maximum exercise}

The evaluation of CO repartition by the LVAD and through the aortic valve can be investigated by combining (invasive) total CO measurement with measured or estimated LVAD pump flow. Acquiring both flows simultaneously is challenging and was done very sparsely in previous clinical studies. Even though crucial insights into pumping performance by the ventricle and the LVAD were missing, the low $\mathrm{pVO}_{2}$ values in LVAD patients as well as the high PCWPs measured at peak exercise do hint towards an incapability of the left ventricle together with the LVAD to accommodate higher CO. Additionally, the occurrence of pulmonary decoupling, the decoupling between diastolic pulmonary artery pressure and PCWP, may further complicate the interpretation of hemodynamics during exercise due to its impact on right heart function.

\section{Contribution of native response and LVAD support to exercise}

A clinical observational study of continuous LVAD monitoring shed light on the individual's hemodynamic response during exercise with constant LVAD speed $(29,30)$. The study reported an inadequate increase in
LVAD output (of approximately $+1 \mathrm{~L} / \mathrm{min}$ on average) from baseline to peak exercise. This increase in LVAD output was independent on aortic valve opening at peak exercise. Furthermore, similar increases in LVAD output were observed during submaximal compared to maximal workloads. Therefore, regression analysis of multiple LVAD monitored parameters with $\mathrm{pVO}_{2}$ showed relevant associations of the cardiac response, namely heart rate (HR) reserve and aortic valve opening, whereas increase in LVAD output was irrelevant (29).

In general, the following parameters have been reported to show associations with $\mathrm{pVO}_{2}$ : age, total $\mathrm{CO}$, flow through the aortic valve, peak arterial venous oxygen difference, chronotropic incompetence (HR reserve), hemoglobin levels, PCWP, NT-pro BNP, valve pathology or status, absolute LVAD parameters and timing of LVAD implant (18,31-34). Furthermore, as opposed to stable HF patients where $\mathrm{pVO}_{2} \mathrm{~s}$ are clinically interpreted based on beta blockers, in LVAD patients treatment with beta blockers showed no clear trend of influencing $\mathrm{pVO}_{2} \mathrm{~s}$, nor did atrial fibrillation, noradrenaline levels or HF etiology $(18,32,33,35)$. Contradictory results compared to the aforementioned studies were in regards to device type, chronotropic incompetence as well as determinants of right heart function $(18,31,32,34,35)$.

One possible reason why the LVAD itself may remain as the bottleneck during maximum exercise capacity testing is the insufficient device monitoring capabilities. Continuous LVAD monitoring during exercise would allow assessment of the hemodynamic interactions in terms of aortic and left ventricular differential pressure (pump head). In a clinical study where LVAD waveforms were monitored, different responses to maximal exercise were reported (30). Analysis of these responses with the information of aortic valve opening, or non-opening, helped to understand and stratify patient responses based on the complex interaction between the ventricle, the LVAD and the vascular system.

\section{Submaximal $v s$. maximal exercise}

Predicted values, calculated by percentage to averages of gender and age-matched healthy controls, showed higher values for submaximal than maximal exercise capacity $(18,29,36)$. This might imply that the stress generated by submaximal exercise, where regulatory mechanisms are not involved to their maximum, might be better tolerated for LVAD patients. Furthermore, the correlation of percentage of expected $6 \mathrm{MW}$ distance with the percentage 
of $\mathrm{pVO}_{2}$ could increase the relevancy to perform $6 \mathrm{MW}$ tests (18). The following variables correlated with $6 \mathrm{MW}$ distance were reported: age, HF severity (determined by NYHA functional class and INTERMACS profiles), atrial fibrillation, diabetes, hemoglobin and estimated glomerular filtration rate $(18,37)$. However for the comparison of patients stratified by a $6 \mathrm{MW}$ distance cutoff of $300 \mathrm{~m}$, variables such as age, atrial fibrillation, HF etiology, INTERMACS profile or echocardiographic right ventricular parameters, did not differ between the groups as reported in Hoermandinger et al. (38).

Due to the still unclear picture of maximal and submaximal exercise tests, pre- and/or post-LVAD implantation, further studies have been conducted $(34,39,40)$. Exercise test results and their potential for risk stratification would provide important clinical insights $(17,21,41)$. For possible LVAD device adjustments or changes in medical therapy, exercise test results might be helpful as proposed in Corrà et al. (17). Submaximal tests for these purposes have proven to be relevant (42). Nevertheless, to utilize the recognized benefits of exercise, a regular medical training regime should be performed (43).

\section{Insights from a cardiorespiratory simulator}

To unravel the complexity of exercise physiology with an LVAD, described in the previous paragraphs, a cardiorespiratory simulator was used. Computational simulators representing the entire cardiorespiratory system are usually based on lumped parameters models, meaning that vessels are lumped into compartment with specific resistances, compliances and inertances.

\section{Description of the cardiorespiratory simulator}

In this paper, we describe a cardiorespiratory simulator, specifically tailored to reproduce and investigate exercise hemodynamics in LVAD patients. The simulator was already presented extensively in previous papers $(44,45)$, we will describe it here briefly. The simulator includes the left and right ventricles, represented in terms of a pressurevolume relationship (46). Such a model accounts for the Frank Starling mechanism, thus allowing to simulate the adaptation of ventricular output to the increased venous return during exercise. For atria, a similar model is used, that accounts also for the effect of atrial contraction on ventricular filling. Vessels are represented with Windkessel models, where blood flow is related to blood pressure in a vessel through one or more resistances, a compliance and an inertance. Vessels are grouped together into six main blocks (upper body, kidneys, splanchnic circulation, left and right legs, pulmonary circulation), each including arterial and venous sections (44). In addition, a specific representation of the ascending and descending aorta, superior and inferior vena cava is also provided.

To simulate the evolution of the cardiovascular simulator from rest to exercise, some control mechanisms were added. Indeed, physical activity elicits several adaptation phenomena both at the level of the myocardium and of the vasculature, leading to a progressive increase in oxygen delivery to the exercising regions. A baroreflex model accounts for the control on the heart and peripheral vessels. The model simulates the sympathovagal balance resetting during exercise, resulting in an overstimulation of the sympathetic system and a withdrawal of the vagal system. As such, the heart's positive inotropy and chronotropy and peripheral vasoconstriction for concurrent increasing values of aortic pressure are simulated during exercise. Moreover, a sympatholysis model was also included, that acts in the circulatory regions exhibiting higher metabolic activity. The balance between the baroreflex and metabolic regulations controls blood pressure and $\mathrm{CO}$ during exercise. While the sympathetic model evokes vasoconstriction peripherally preventing hypotension, the metabolic control model induces local vasodilation in the vascular regions requiring more perfusion. Finally, to simulate bicycle exercise (usually used in cardiopulmonary tests in LVAD patients), a muscle contraction model was added to the legs, that produces a pressure outside the vessels proportional to the cycling load (44).

Since exercise ultimately translates into higher oxygen delivery to the body, a model of the respiratory system was also implemented. The respiratory model contains three main modules: ventilation mechanics, ventilation control and gas exchange in the alveoli and peripheral tissues. The ventilation mechanics model describes breathing activity and was implemented through a resistive element for the airways and an elastance element for the lungs. The resulting intrathoracic pressure affects the vessels within the chest and in turn the venous return. The ventilation control adapts the ventilated flow according to the levels of oxygen and carbon dioxide sensed in the simulated upper body. For gas exchange, mass balance equations were implemented. In the alveoli, the equation accounts for the amount of inspired and expired air at each ventilation cycle and for the pulmonary flow. In the tissues, the equation accounts for 
the arterial blood inflow and venous blood outflow and the metabolic activity in the considered region.

A block diagram of the cardiorespiratory simulator described so far is reported in Figure 1.

The cardiorespiratory simulator was tuned to the condition of an average LVAD patient. It is known that an LVAD is not capable to completely reverse the underlying $\mathrm{HF}$ condition of a patient and that many impairments persist after LVAD implantation at the level of both the cardiovascular and respiratory systems (24).

A detailed description on how to tune cardiovascular parameters to an average LVAD patient is provided in Fresiello et al. (45). Briefly, systolic and diastolic functions are adjusted to mimic the mechanical properties of ventricles. In LVAD patients, the contractility of the left (and in some cases of the right) ventricle is impaired compared to a healthy heart. Concerning diastole, the left ventricle is stiffer than a failed unassisted ventricle, due to the "reverse-remodelling" operated by the LVAD (47). The systemic and pulmonary circulation are adjusted to reproduce a relatively lower peripheral resistance [due to pharmacological treatment (48)] and a slightly higher pulmonary resistance, observed in some LVAD patients (49). In addition, some tuning of the control mechanisms that drive the adaptation of the cardiovascular system to exercise are also performed (44). HF patients are chronically exposed to low values of perfusion, it is likely to assume that tissues adapted to poorer values of oxygen content. Therefore, the metabolic control model is tuned to be less efficient in inducing vasodilation. Also, a sympathovagal imbalance is implemented to reproduce the chronotropic incompetence often observed in LVAD patients (18). Finally, the respiratory system embeds some impairments related to the HF condition: a reduced perfusion of ventilated lungs and an increased respiratory quotient (50).

Concerning the LVAD model, a rotary blood pump can be represented hemodynamically by an equation relating pump flow, pressure head and impeller speed (51):

$$
\begin{aligned}
\Delta P(t)= & \alpha_{L V A D} \cdot Q_{L V A D}(t)^{2}+\beta_{L V A D}-R_{L V A D} \cdot Q_{L V A D}(t) \\
& -L_{L V A D} \cdot \frac{d Q_{L V A D}(t)}{d t}
\end{aligned}
$$

Where $\Delta P$ is the pressure difference across the LVAD (pump head), $Q_{L V A D}$ is the LVAD flow, $R_{L V A D}$ and $L_{L V A D}$ are the resistance and the inertance of LVAD, $\alpha_{L V A D}$ and $\beta_{\text {LVAD }}$ are parameters that depend on pump pressure-flow characteristics and the selected rotational speed. Additional resistive and inertial elements can be added to the equation to represent the inflow cannula and outflow graft (52).

The simulator was validated at rest and at exercise for healthy, HF and LVAD patients $(44,45)$. Once validated, the simulator was used to unravel the complexity of exercise physiology in presence of an LVAD. Indeed, the simulator offers the opportunity to rationalize the physiological phenomena taking place during physical activity and to investigate the impact of a single parameter on general hemodynamics. Specifically, three main topics were investigated and discussed hereafter.

\section{What's the impact of the LVAD on exercise bemodynamics?}

To answer this question, an average LVAD patient was simulated at rest, supported by an HVAD (Medtronic Inc., Minneapolis, MN, USA) running at 2,700 rpm. Then, a bicycle exercise of 80 Watts of intensity was imposed on the simulator, corresponding to an oxygen uptake of $15.2 \mathrm{~mL} / \mathrm{min} / \mathrm{kg}$ [average peak value reached by LVAD patients (19)]. Simulation output was compared with hemodynamic data of LVAD patients at rest and at maximal exercise taken from the literature (19,53-56) (see Table 1).

According to simulations, LVAD patients experience an increase in wedge pressure from rest to exercise. The increased wedge pressure indicates on one hand an insufficient capacity of the LVAD and the native ventricle to cope with the right ventricular output, on the other hand an insufficient capacity of the native ventricle to accommodate increased blood volumes.

For a better explanation, the simulated pressure-volume loops of the assisted ventricle are shown in Figure 2. They are compared with the pressure-volume loops simulated for a failed unassisted ventricle (HF) at rest and when cycling at an intensity of 56 Watts. The LVAD provokes a "reverse remodelling" of the failed ventricle chronically that consists in stiffer elastic properties in diastole. Therefore, the assisted ventricle is smaller and works on a nonlinear portion of the diastolic curve, while the HF ventricle is larger and works in a more linear portion of the diastolic curve. During exercise, venous return increases. In the HF ventricle, contractility increases very little or not at all, so the ventricle resorts to only using the Frank Starling mechanism: the end diastolic volume increases in order for the ventricle to eject a larger stroke volume. In the assisted ventricle the increase of volume simulated is less evident, in agreement with $(54,55)$. But because of the diastolic stiffness, even a small increase in volume translates in a 

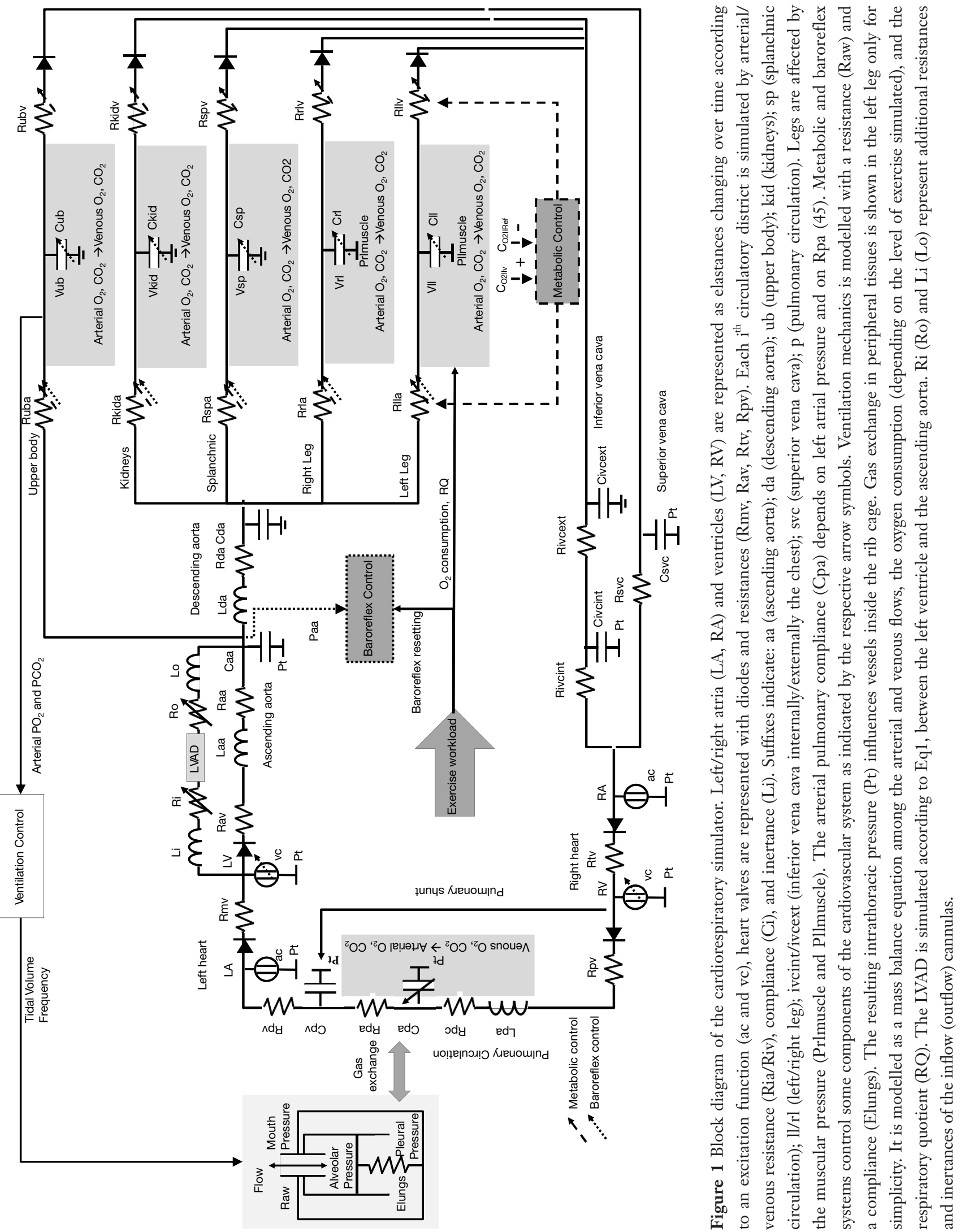
Table 1 Comparison between the data of LVAD patients taken from clinical studies (19,53-56) and the simulated data of an average patient with an HVAD support of 2,700 rpm. Data refer to the hemodynamic condition at rest and at maximal exercise

\begin{tabular}{|c|c|c|c|c|}
\hline Variable & \multicolumn{2}{|l|}{ Rest } & \multicolumn{2}{|l|}{ Exercise } \\
\hline Heart rate, bpm & $76 \pm 3$ & 75 & $117 \pm 15$ & 113 \\
\hline Cardiac output, L/min & $5.0 \pm 0.4$ & 4.8 & $7.3 \pm 1.4$ & 6.8 \\
\hline Aortic pressure, $\mathrm{mmHg}$ & $90.6 \pm 8.8$ & 92.1 & $98.3 \pm 12.8$ & 100.5 \\
\hline Wedge pressure, $\mathrm{mmHg}$ & $15.1 \pm 2.7$ & 13.2 & $22.3 \pm 3.1$ & 25.5 \\
\hline Right atrial pressure, $\mathrm{mmHg}$ & $7.4 \pm 1.4$ & 8.7 & $13.0 \pm 4.0$ & 16.5 \\
\hline LVES, mL & 160 & 149 & 154 & 156 \\
\hline LVED, mL & $193 \pm 16$ & 179 & $186 \pm 18$ & 194 \\
\hline
\end{tabular}

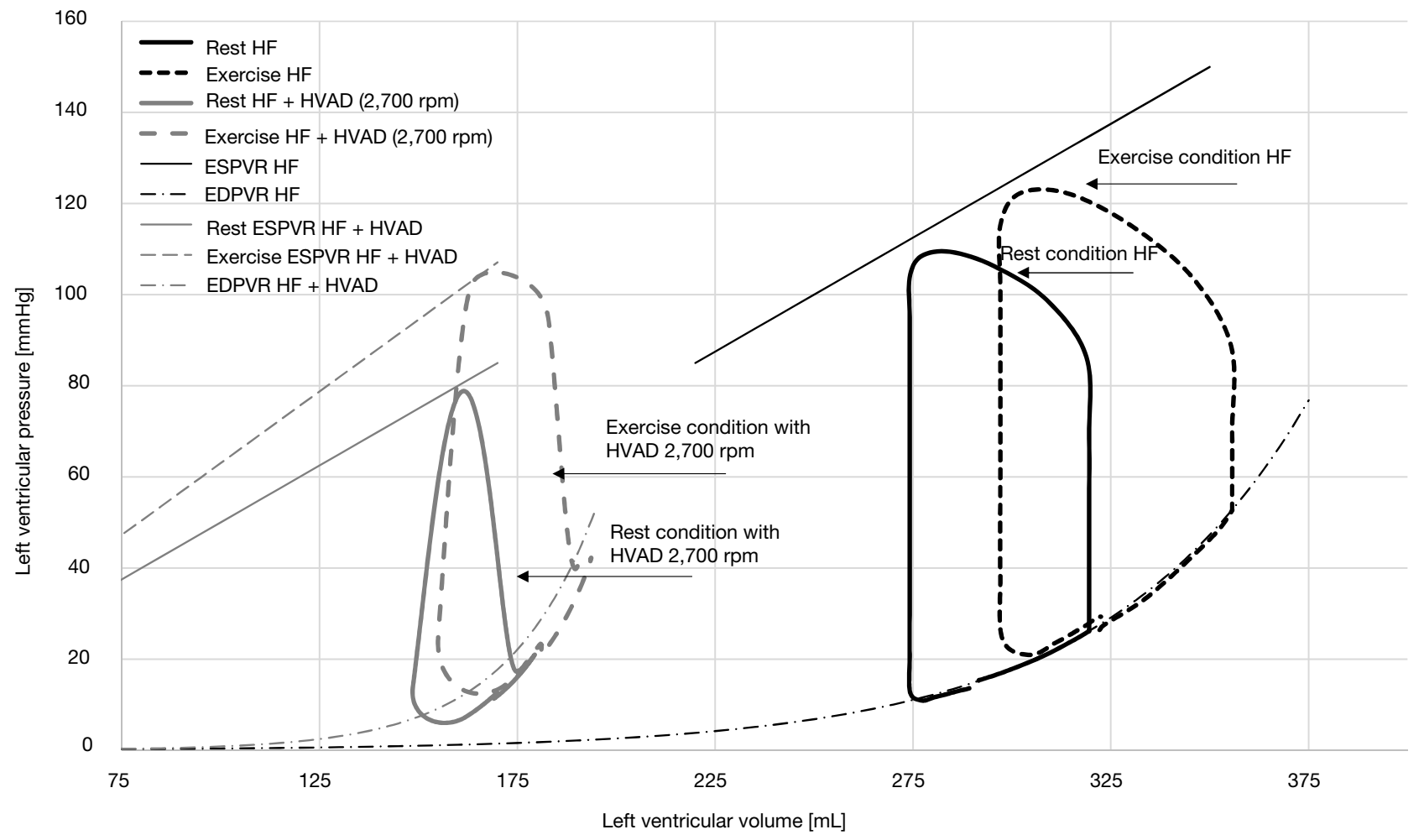

Figure 2 Simulated pressure-volume loops of the left ventricle in: heart failure condition (HF) at rest and during cycling at 56 Watts, in heart failure supported by a HVAD at 2,700 rpm at rest and during cycling at 80 Watts. The plot includes the end-diastolic pressure volume relationship (EDPVR) and the end-systolic pressure volume relationship (ESPVR) curves. 
large increase in pressure.

Concerning CO, simulations in Table 1 show that at peak exercise $\mathrm{CO}$ increases of $+2.0 \mathrm{~L} / \mathrm{min}$, a change comparable to that observed in HF patients (56-58), and lower than that of healthy subjects (59). The increase in $\mathrm{CO}$ is the combined effect of the LVAD flow increase $(+0.5 \mathrm{~L} / \mathrm{min})$ and of the native ventricle that starts to eject at exercise $(+1.5 \mathrm{~L} / \mathrm{min})$. This result is in agreement with Gross et al. (29) observing a higher likelihood of aortic valve opening at exercise than at rest. Given the modest increase of $\mathrm{CO}$ and the limited contribution of the LVAD, in the next paragraph we investigated the possible hemodynamic benefits of an increase in LVAD speed during exercise.

\section{What's the impact of LVAD speed increase on exercise hemodynamics?}

To answer this question, we started from the simulated average patient with an HVAD support of 2,700 rpm performing a bicycle exercise of 80 Watts intensity (described in Table 1). Starting from this average patient (average), additional exercise patient profiles were simulated. For this purpose, some parameters of the simulator were released from the controls and manually imposed systematically: left/right systolic function (Emaxl/ Emaxr), HR, and systemic resistance [total peripheral resistance (TPR)]. These parameters were changed individually by $\pm 20 \%$, compared to the value of average. This allowed us to simulate a patient with a better/poorer inotropic, chronotropic and vascular response to exercise. Additionally, profiles with aortic stenosis (AS) and aortic insufficiency (AI) were also simulated.

Each profile was simulated at peak exercise with a baseline HVAD speed of 2,700 rpm and with increased HVAD speed of 3,500 rpm. Results are reported in Figure 3, where the $\mathrm{CO}$ is plotted for each profile at the HVAD speed of 2,700 rpm. The increase of $\mathrm{CO}$ due to the augmented HVAD speed is plotted in addition.

For the average patient, $\mathrm{CO}$ is $6.8 \mathrm{~L} / \mathrm{min}$ at exercise, of which $1.5 \mathrm{~L} / \mathrm{min}$ is provided by the native ventricle. LVAD speed increase produces an additional $+1.0 \mathrm{~L} / \mathrm{min}$ of $\mathrm{CO}$, in agreement with Mezzani et al. (60). This higher LVAD speed provokes an augmented LVAD output that ultimately sets the ventricle back to full support. The patient profile with increased left ventricular contractility (Emaxl+20\%) has already better $\mathrm{CO}$ at exercise for baseline speed $(7.4 \mathrm{~L} / \mathrm{min})$, of which $2.7 \mathrm{~L} / \mathrm{min}$ is pumped by the ventricle. The increased LVAD speed elicits a moderate improvement of
$\mathrm{CO}(+0.8 \mathrm{~L} / \mathrm{min})$, since it ultimately results in a competition between the left ventricle and the LVAD in ejecting blood. Indeed, the native ventricle with a better residual contractility still ejects for a concomitant LVAD speed increase during exercise. This ventricular output provokes a reduced sensitivity of LVAD to preload compared to a full support condition [for more info please refer to (29)]. Conversely, the Emaxl-20\% profile benefits from an LVAD speed increase the most $(\mathrm{CO}+1.6 \mathrm{~L} / \mathrm{min})$. At baseline speed, the Emaxl-20\% profile shows very little ventricular output $(0.2 \mathrm{~L} / \mathrm{min})$. At a higher speed, the LVAD completely overcomes the ventricle in ejecting blood. Patients profiles with a poorer chronotropic response (HR-20\%) and with poorer vascular response $(\mathrm{TPR}+20 \%)$ evidence major $\mathrm{CO}$ improvement with higher LVAD speed $(+1.2,1.3 \mathrm{~L} / \mathrm{min}$ respectively) compared to their counterparts $(+0.9 \mathrm{~L} / \mathrm{min}$ for $\mathrm{HR}+20 \%$ and $+1.1 \mathrm{~L} / \mathrm{min}$ for TPR $-20 \%$ ). For Emaxr+20\% and Emaxr-20\%, the increase of CO due to LVAD speed increase is comparable $(+0.9 \mathrm{~L} / \mathrm{min}$ and $+1.1 \mathrm{~L} / \mathrm{min}$, respectively). Finally, for a patient with AS, $\mathrm{LVAD}$ increase can bring $+1.2 \mathrm{~L} / \mathrm{min}$ to $\mathrm{CO}$. In case of aortic regurgitation $\mathrm{CO}$ increase is more modest $(+0.7 \mathrm{~L} / \mathrm{min})$ and at the expenses of the left ventricle that has to pump regurgitant flow additionally $(-1.3 \mathrm{~L} / \mathrm{min}$ in total).

Simulations showed that the benefits of LVAD speed increase depend on patients' underlying condition and cardiovascular impairments. These results can explain in part the different and sometimes contradictory results reported by clinical studies investigating a manual LVAD speed increase during maximal cardiopulmonary exercise tests (19,24-28). In most of these studies, LVAD speed was increased mildly or moderately and of the same amount regardless the type of patient. According to our simulations, patients with a poorer chronotropic, inotropic and vascular response, namely older HF patients, are likely to benefit more from an LVAD speed controller, as observed clinically in (28).

Concerning the wedge pressure, for the simulated profiles with increased LVAD speed values ranged from $17.1 \mathrm{mmHg}$ (Emaxr-20\%) to $24.2 \mathrm{mmHg}$ (Emaxr+20\%). It is evident that the LVAD is unable to properly accommodate and pump forward the increased preload, even after a substantial speed increase (as 3,500 rpm for the HVAD is already beyond the limits sets for clinical use). Hence, there could be benefits in a further upregulation in LVAD speed in certain patients or an LVAD with a larger output range than what is currently clinically available. It is worth noting that in healthy subjects, $\mathrm{CO}$ increases at least 3 -fold to 


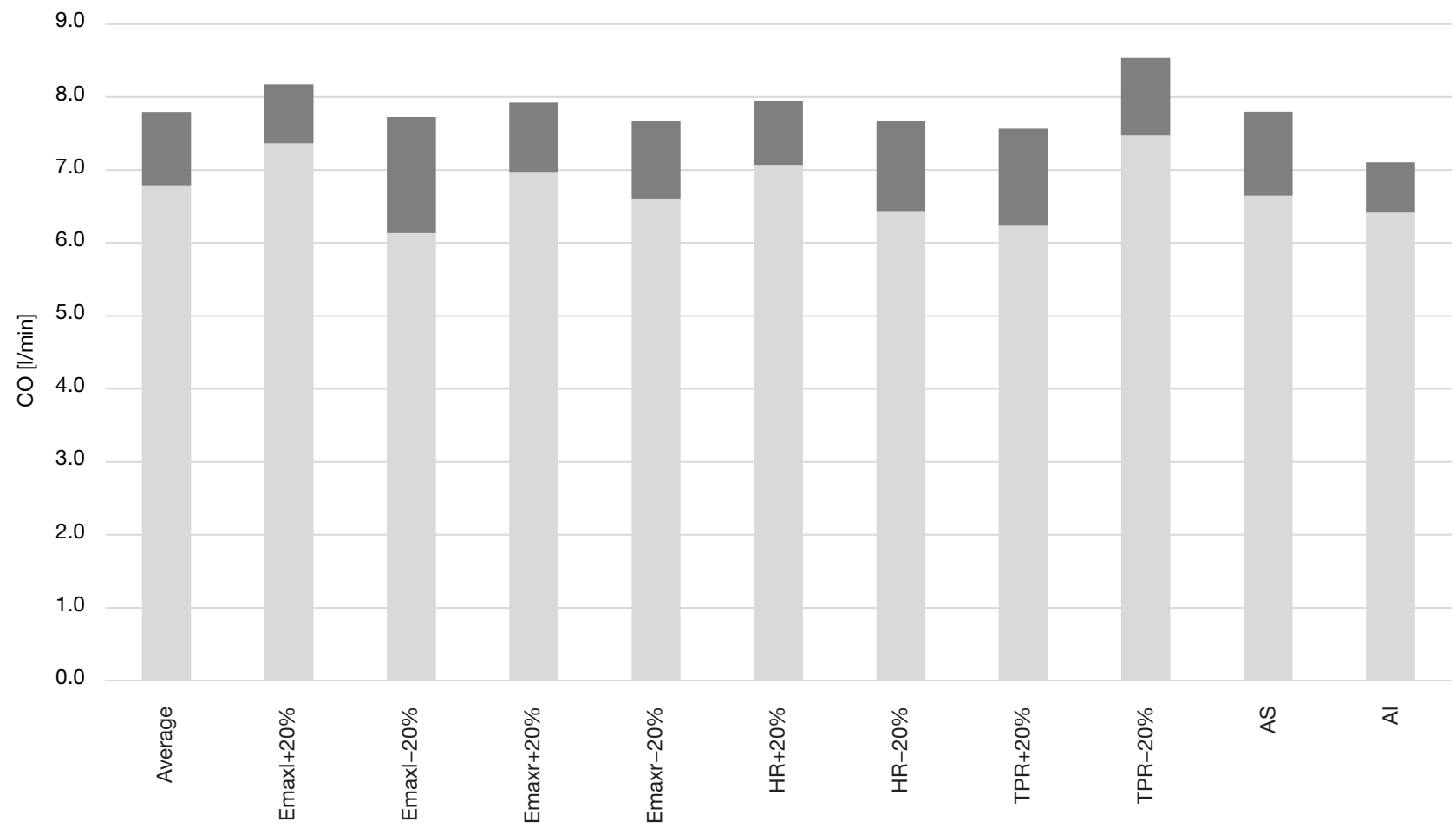

Figure 3 Simulated cardiac output (CO) for different profiles of left ventricular assist device (LVAD) patients during cycling at 80 Watts. Light grey columns refer CO each patient profile would reach with a HVAD speed of 2,700 rpm; dark grey columns indicate the additional CO achievable by increasing HVAD speed up to 3,500 rpm. Baseline refers to the average patient profile, Emaxl+20\% (-20\%) refers to a patient with a better (poorer) left inotropic response at exercise, Emaxr+20\% (-20\%) refers to a patient with a better (poorer) right inotropic response at exercise, $\mathrm{HR}+20 \%(-20 \%)$ refers to a patient with a better (poorer) right chronotropic response at exercise, TPR+20\% (-20\%) refers to a patient with a lower (higher) peripheral vasodilation at exercise, AS (AI) refer to a patient profile with aortic stenosis (regurgitation). $\mathrm{HR}$, heart rate; TPR, total peripheral resistance; AS, aortic stenosis; AI, aortic insufficiency.

$15-20 \mathrm{~L} / \mathrm{min}$ during physical activity, values far beyond the capabilities of current LVADs.

\section{What's the impact of LVAD pressure-flow curves on exercise hemodynamics?}

Current LVADs operate at a constant impeller speed and are unable to regulate pump flow to body demands. As such, $\mathrm{Q}_{\mathrm{LVAD}}$ ultimately depends on pump head $\Delta \mathrm{P}$ and on the pressure-flow curves that can change according to pump sped and inner design \{see Eq. [1]\}. An LVAD with a flatter pressure-flow curve is defined as "pressure sensitive", namely a small decrease in $\Delta \mathrm{P}$ produces a large increase in pump flow. Two examples of pressure-flow curve, for a centrifugal and an axial pump (LVAD1 and LVAD2, respectively) are reported in Figure 4.

Given these premises, the question is which type of
LVAD pressure-flow curve would better support exercise hemodynamics (here we assume no LVAD speed control in place, so a constant speed is set for the simulations).

To answer this question, we first need to understand how LVAD flow profile evolves from rest to exercise due to the surrounding hemodynamics across the pump. In a previous paper, the authors investigated this topic in 14 patients with an HVAD that performed 24 maximal bicycle exercise tests (30). Again, the interaction between the LVAD and the cardiovascular system was shown to be patient specific. LVAD flow features (average, diastolic, systolic values and pulsatility) evolved differently from rest to exercise according to patients. In some cases, an overall increase of all LVAD flow features was observed, in other cases a decrease of LVAD flow in diastole or a decrease in flow pulsatility were reported [the underlying cardiovascular reasons for the different evolutions are reported in (30)]. 


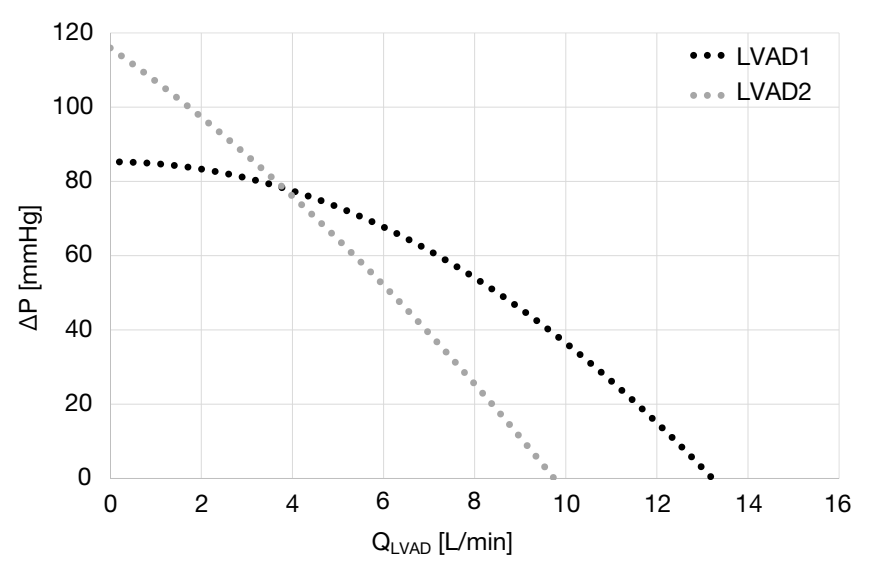

Figure 4 Simulated stationary pressure-flow curves of LVAD1 and LVAD2 excluding cannulation. Black line: pressure-flow curve similar to a centrifugal LVAD under development at ReinVAD $\mathrm{GmbH}$, Germany. Grey line: pressure-flow curve similar to the axial HeartMate II (Abbott Laboratories, USA). The black line describes a more pressure sensitive pump than the grey line. LVAD, left ventricular assist device.

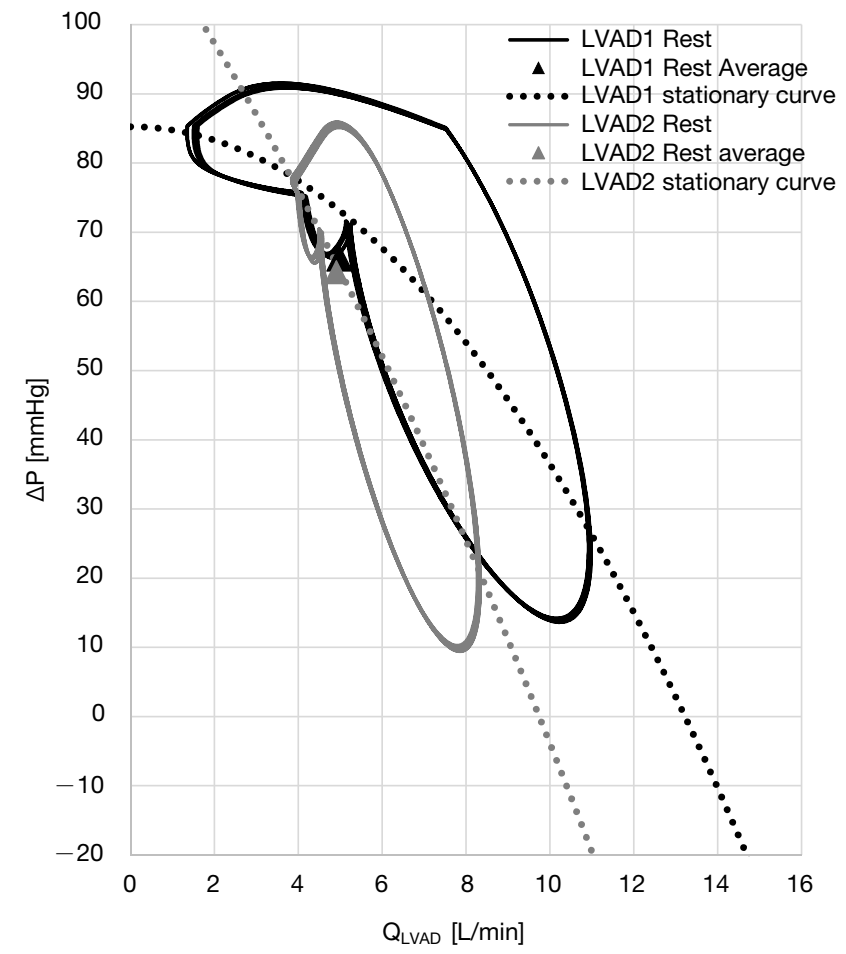

Most cases showed a general increase in all LVAD flow features, in agreement with our simulated average patient described beforehand. Starting from this profile, two pumps with the pressure-flow characteristics reported in Figure 4 were simulated at rest and at bicycle exercise of 80 Watts intensity. Both LVADs provide same average LVAD flow at rest of $4.9 \mathrm{~L} / \mathrm{min}$, but each $\mathrm{LVAD}$ is expected to react differently to the $\Delta \mathrm{P}$ changes at exercise according to the pump-specific pressure-flow characteristics.

From Figure 5-left panel we can observe that the dynamic pressure-flow curves differ for LVAD1 and LVAD2, but the average $\mathrm{Q}_{\mathrm{LVAD}}$ value is comparable for both pumps. As such, both pumps provide the same level of support at rest. During exercise (Figure 5-right panel) a right down-shift of the LVAD pressure-flow loops is observed. Indeed, the $\triangle \mathrm{P}$ across the LVAD is reduced both in diastole (due to the increase of wedge pressure discussed beforehand), and in systole (since the left ventricle starts to eject). Because of the flatter pressure-flow characteristics, the more "pressure sensitive" LVAD1 reacts to this $\triangle \mathrm{P}$ drop with a higher

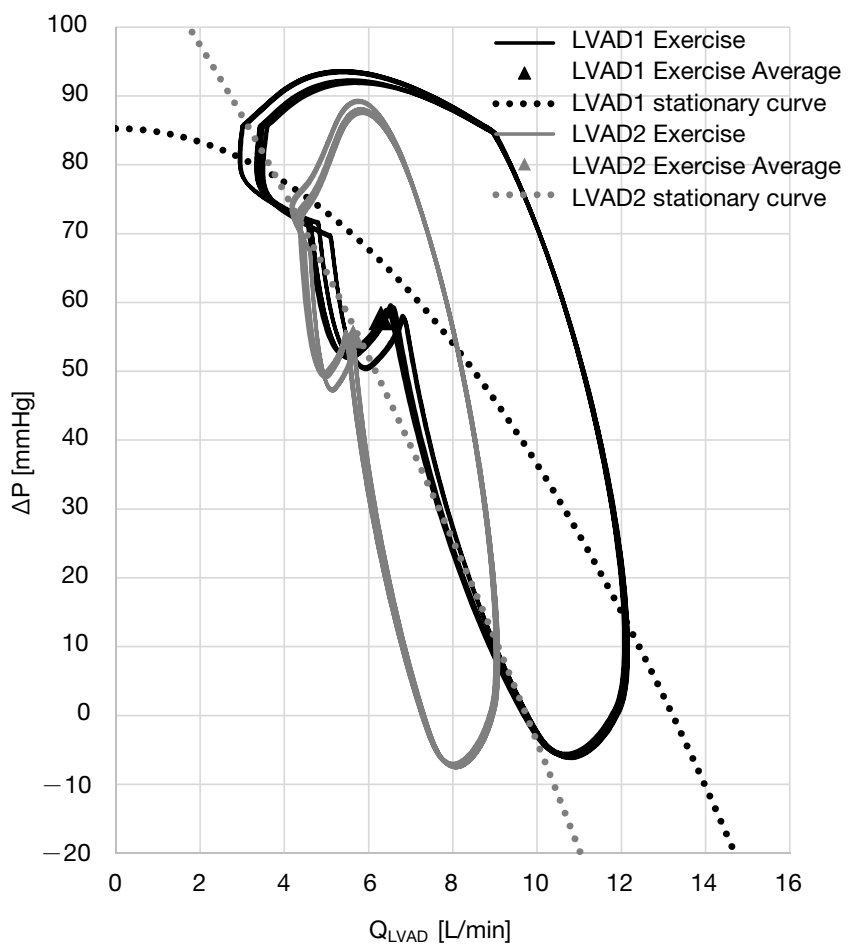

Figure 5 Simulated LVAD pressure-flow curves. Continuous lines: dynamic pressure-flow curves for LVAD1 and LVAD2 at rest (left panel) and at exercise (right panel). The shown hysteresis runs counter-clockwise and is due to the inertia in the respective pump and cannulas. Dotted lines: stationary pressure-flow curves for LVAD1 and LVAD2. The triangles illustrate the average $\mathrm{Q}_{\mathrm{LVAD}}$ and $\triangle \mathrm{P}$ values over heart cycles. LVAD, left ventricular assist device. 
increase in flow (average $\mathrm{Q}_{\mathrm{LVAD}}$ is $6.3 \mathrm{~L} / \mathrm{min}$ for LVAD1 and 5.6 L/min for LVAD2). As such, LVAD1 can better support hemodynamics in patients' profiles, experiencing an overall reduction of load across the pump at exercise.

\section{Conclusions}

Exercise limitation in LVAD patients is a multifactorial problem, due to a non-complete reversing of the underlying HF condition.

In this complex and diverse scenario, the contribution of cardiovascular modelling is to offer a comprehensive and rationalized representation of the variables involved in exercise physiology. The simulator presented here reproduced the cardiorespiratory system and the adaptation mechanisms occurring from rest to exercise with their major impairments due to HF. The simulator reproduced the hemodynamic limitations in LVAD patients at exercise and the change of working conditions for the native ventricle. Simulation results showed that flatter pressure-flow characteristics can better sustain patients' hemodynamics. Concerning LVAD speed increase and its possible benefits on exercise, a comparative study was conducted where different patients' profiles were simulated to account for the hemodynamic diversity observed in the clinics. The resulting testing scenarios revealed the need for different LVAD speed strategies, tailored on patients' specific vascular and cardiac conditions. Such a simulator can be used to define and test physiological control algorithms for LVADs as well as to define new therapeutic strategies aimed at improving patients' exercise responses.

\section{Acknowledgments}

Funding: This work was supported by the KUL internal grant C14/18/080.

\section{Footnote}

Conflicts of Interest: The authors have no conflicts of interest to declare.

Open Access Statement: This is an Open Access article distributed in accordance with the Creative Commons Attribution-NonCommercial-NoDerivs 4.0 International License (CC BY-NC-ND 4.0), which permits the noncommercial replication and distribution of the article with the strict proviso that no changes or edits are made and the original work is properly cited (including links to both the formal publication through the relevant DOI and the license). See: https://creativecommons.org/licenses/by-nc-nd/4.0/.

\section{References}

1. Kirklin JK, Xie R, Cowger J, et al. Second annual report from the ISHLT Mechanically Assisted Circulatory Support Registry. J Heart Lung Transplant 2018;37:685-91.

2. Kormos RL, Cowger J, Pagani FD, et al. The Society of Thoracic Surgeons Intermacs database annual report: Evolving indications, outcomes, and scientific partnerships. J Heart Lung Transplant 2019;38:114-26.

3. Lim HS, Howell N, Ranasinghe A. The Physiology of Continuous-Flow Left Ventricular Assist Devices. J Card Fail 2017;23:169-80.

4. Tsiouris A, Heliopoulos I, Mikroulis D, et al. Factors defining occurrence of ischemic and hemorrhagic strokes during continuous flow left ventricular assist device support. Gen Thorac Cardiovasc Surg 2020;68:319-27.

5. Sequeira $V$, van der Velden J. Historical perspective on heart function: the Frank-Starling Law. Biophys Rev 2015;7:421-47.

6. Imamura T, Chung B, Nguyen A, et al. Clinical implications of hemodynamic assessment during left ventricular assist device therapy. J Cardiol 2018;71:352-8.

7. Mikus E, Stepanenko A, Krabatsch T, et al. Reversibility of fixed pulmonary hypertension in left ventricular assist device support recipients. Eur J Cardiothorac Surg 2011;40:971-7.

8. Biełka A, Kalinowski M, Hawranek M, et al. Mechanical circulatory support restores eligibility for heart transplant in patients with significant pulmonary hypertension. Kardiol Pol 2020;78:1008-14.

9. Yalcin YC, Muslem R, Veen KM, et al. Impact of Continuous Flow Left Ventricular Assist Device Therapy on Chronic Kidney Disease: A Longitudinal Multicenter Study. J Card Fail 2020;26:333-41.

10. Deo SV, Sharma V, Altarabsheh SE, et al. Hepatic and renal function with successful long-term support on a continuous flow left ventricular assist device. Heart Lung Circ 2014;23:229-33.

11. Estep JD, Starling RC, Horstmanshof DA, et al. Risk assessment and comparative effectiveness of left ventricular assist device and medical management in ambulatory heart failure patients results from the ROADMAP Study. J Am Coll Cardiol 2015;66:1747-61.

12. Kirklin JK, Pagani FD, Kormos RL, et al. Eighth annual 
INTERMACS report: Special focus on framing the impact of adverse events. J Heart Lung Transplant 2017;36:1080-6.

13. Jakovljevic DG, McDiarmid A, Hallsworth K, et al. Effect of left ventricular assist device implantation and heart transplantation on habitual physical activity and quality of life. Am J Cardiol 2014;114:88-93.

14. Emin A, Rogers CA, Banner NR, et al. Quality of life of advanced chronic heart failure: Medical care, mechanical circulatory support and transplantation. Eur J Cardiothorac Surg 2016;50:269-73.

15. Teuteberg JJ, Cleveland JC, Cowger J, et al. The Society of Thoracic Surgeons Intermacs 2019 Annual Report: The Changing Landscape of Devices and Indications. Ann Thorac Surg 2020;109:649-60.

16. Albouaini K, Egred M, Alahmar A, et al. Cardiopulmonary exercise testing and its application. Postgrad Med J 2007;83:675-82.

17. Corrà U, Agostoni PG, Anker SD, et al. Role of cardiopulmonary exercise testing in clinical stratification in heart failure. A position paper from the Committee on Exercise Physiology and Training of the Heart Failure Association of the European Society of Cardiology: Cardiopulmonary exercise testing and prognosis in HF. Eur J Heart Fail 2018;20:3-15.

18. Fresiello L, Jacobs S, Timmermans P, et al. Limiting factors of peak and submaximal exercise capacity in LVAD patients. PLoS One 2020;15:e0235684.

19. Jung MH, Gustafsson F. Exercise in heart failure patients supported with a left ventricular assist device. J Heart Lung Transplant 2015;34:489-96.

20. Dunlay SM, Allison TG, Pereira NL. Changes in Cardiopulmonary Exercise Testing Parameters Following Continuous Flow Left Ventricular Assist Device Implantation and Heart Transplantation. J Card Fail 2014;20:548-54.

21. Dorken Gallastegi A, Öztürk P, Demir E, et al. Prospective evaluation of ventricular assist device risk scores' capacity to predict cardiopulmonary exercise parameters. Interact Cardiovasc Thorac Surg 2020;30:223-8.

22. Moreno-Suarez I, Liew S, Dembo LG, et al. Physical Activity Is Higher in Patients with Left Ventricular Assist Device Compared with Chronic Heart Failure. Med Sci Sports Exerc 2020;52:1-7.

23. Martina J, de Jonge N, Rutten M, et al. Exercise hemodynamics during extended continuous flow left ventricular assist device support: the response of systemic cardiovascular parameters and pump performance. Artif Organs 2013;37:754-62.

24. Loyaga-Rendon RY, Plaisance EP, Arena R, et al. Exercise physiology, testing, and training in patients supported by a left ventricular assist device. J Heart Lung Transplant 2015;34:1005-16.

25. Schmidt T, Bjarnason-Wehrens B, Schulte-Eistrup S, et al. Effects of pump speed changes on exercise capacity in patients supported with a left ventricular assist device-an overview. J Thorac Dis 2018;10:S1802-10.

26. Hayward CS, Fresiello L, Meyns B. Exercise physiology in chronic mechanical circulatory support patients: vascular function and beyond. Curr Opin Cardiol 2016;31:292-8.

27. Noor MR, Bowles C, Banner NR. Relationship between pump speed and exercise capacity during HeartMate II left ventricular assist device support: influence of residual left ventricular function. Eur J Heart Fail 2012;14:613-20.

28. Jung MH, Hansen PB, Sander K, et al. Effect of increasing pump speed during exercise on peak oxygen uptake in heart failure patients supported with a continuous-flow left ventricular assist device. A double-blind randomized study: Effect of increasing pump speed during exercise. Eur J Heart Fail 2014;16:403-8.

29. Gross C, Marko C, Mikl J, et al. LVAD Pump Flow Does Not Adequately Increase With Exercise. Artif Organs 2019;43:222-8.

30. Gross C, Fresiello L, Schlöglhofer T, et al. Hemodynamic exercise responses with a continuousflow left ventricular assist device: Comparison of patients' response and cardiorespiratory simulations. PLoS One 2020;15:e0229688.

31. Moss N, Rakita V, Lala A, et al. Hemodynamic Response to Exercise in Patients Supported by Continuous Flow Left Ventricular Assist Devices. JACC Heart Fail 2020;8:291-301.

32. Mirza KK, Jung MH, Sigvardsen PE, et al. Computed Tomography-Estimated Right Ventricular Function and Exercise Capacity in Patients with Continuous-Flow Left Ventricular Assist Devices: ASAIO J 2020;66:8-16.

33. Mirza KK, Cuomo K, Jung MH, et al. Effect of Heart Rate Reserve on Exercise Capacity in Patients Treated with a Continuous Left Ventricular Assist Device. ASAIO J 2020;66:160-5.

34. Kondo T, Okumura T, Oishi H, et al. Associations between hemodynamic parameters at rest and exercise capacity in patients with implantable left ventricular assist devices. Int J Artif Organs 2021;44:174-80. 
35. Grosman-Rimon L, Kachel E, McDonald MA, et al. Association Between Neurohormone Levels and Exercise Testing Measures in Patients with Mechanical Circulatory Supports. ASAIO J 2020;66:875-80.

36. Schmidt T, Bjarnason-Wehrens B, Mommertz S, et al. Changes in Total Cardiac Output and Oxygen Extraction During Exercise in Patients Supported With an HVAD Left Ventricular Assist Device. Artif Organs 2018;42:686-94.

37. Gustafsson F, Mirza KK, Pya Y, et al. Predictors of Physical Capacity 6 Months After Implantation of a Full Magnetically Levitated Left Ventricular Assist Device: An Analysis from the ELEVATE Registry. J Card Fail 2020;26:580-7.

38. Hoermandinger C, Krastev H, Mulzer J, et al. Right Ventricular Function Does Not Significantly Influence 6-Minute Walk Distance in Patients with LVAD. J Heart Lung Transplant 2020;39:S340.

39. Koshy A, Green T, Toms A, et al. The role of exercise hemodynamics in assessing patients with chronic heart failure and left ventricular assist devices. Expert Rev Med Devices 2019;16:891-8.

40. Laoutaris ID. Restoring pulsatility and peakVO2 in the era of continuous flow, fixed pump speed, left ventricular assist devices: 'A hypothesis of pump's or patient's speed?'. Eur J Prev Cardiol 2019;26:1806-15.

41. Agostoni P, Paolillo S, Mapelli M, et al. Multiparametric prognostic scores in chronic heart failure with reduced ejection fraction: a long-term comparison: Multiparametric prognostic scores in $\mathrm{HF}$ with reduced ejection fraction. Eur J Heart Fail 2018;20:700-10.

42. Giannitsi S, Bougiakli M, Bechlioulis A, et al. 6-minute walking test: a useful tool in the management of heart failure patients. Ther Adv Cardiovasc Dis 2019;13:1753944719870084.

43. Marko C, Xhelili E, Lackner T, et al. Exercise Performance During the First Two Years After Left Ventricular Assist Device Implantation: ASAIO J 2017;63:408-13.

44. Fresiello L, Meyns B, Di Molfetta A, et al. A Model of the Cardiorespiratory Response to Aerobic Exercise in Healthy and Heart Failure Conditions. Front Physiol 2016;7:189.

45. Fresiello L, Rademakers F, Claus P, et al. Exercise physiology with a left ventricular assist device: Analysis of heart-pump interaction with a computational simulator. PloS One 2017;12:e0181879.

46. Sagawa K, Maughan L, Suga H, et al. Cardiac contraction and the Pressure- Volume relationship. 1st edition. New York: Oxford University Press, 1988.

47. Marinescu KK, Uriel N, Mann DL, et al. Left ventricular assist device-induced reverse remodeling: it's not just about myocardial recovery. Expert Rev Med Devices 2017;14:15-26.

48. Bennett MK, Adatya S. Blood pressure management in mechanical circulatory support. J Thorac Dis 2015;7:2125-8.

49. Krishnamurthy Y, Cooper LB, Parikh KS, et al. Pulmonary Hypertension in the Era of Mechanical Circulatory Support. ASAIO J 2016;62:505-12.

50. Wasserman K, Zhang YY, Gitt A, et al. Lung function and exercise gas exchange in chronic heart failure. Circulation 1997;96:2221-7.

51. Moscato F, Danieli GA, Schima H. Dynamic modeling and identification of an axial flow ventricular assist device. Int J Artif Organs 2009;32:336-43.

52. Graefe R, Henseler A, Körfer R, et al. Influence of left ventricular assist device pressure-flow characteristic on exercise physiology: Assessment with a verified numerical model. Int J Artif Organs 2019;42:490-9.

53. Jakovljevic DG, George RS, Nunan D, et al. The impact of acute reduction of continuous-flow left ventricular assist device support on cardiac and exercise performance. Heart 2010;96:1390-5.

54. Brassard P, Jensen AS, Nordsborg N, et al. Central and Peripheral Blood Flow During Exercise with a Continuous-Flow Left Ventricular Assist Device: Constant Versus Increasing Pump Speed: A Pilot Study. Circ Heart Fail 2011;4:554-60.

55. Muthiah K, Robson D, Prichard R, et al. Effect of exercise and pump speed modulation on invasive hemodynamics in patients with centrifugal continuous-flow left ventricular assist devices. J Heart Lung Transplant 2015;34:522-9.

56. Jacquet L, Vancaenegem O, Pasquet A, et al. Exercise capacity in patients supported with rotary blood pumps is improved by a spontaneous increase of pump flow at constant pump speed and by a rise in native cardiac output. Artif Organs 2011;35:682-90.

57. Kirlin PC, Das S, Zijnen P, et al. The exercise response in idiopathic dilated cardiomyopathy. Clin Cardiol 1984;7:205-10.

58. Mancini D, Goldsmith R, Levin H, et al. Comparison of exercise performance in patients with chronic severe heart failure versus left ventricular assist devices. Circulation 1998;98:1178-83. 
59. Sullivan MJ, Knight JD, Higginbotham MB, et al. Relation between central and peripheral hemodynamics during exercise in patients with chronic heart failure. Muscle blood flow is reduced with maintenance of arterial perfusion pressure. Circulation 1989;80:769-81.

Cite this article as: Fresiello L, Gross C, Jacobs S. Exercise physiology in left ventricular assist device patients: insights from hemodynamic simulations. Ann Cardiothorac Surg 2021;10(3):339-352. doi: 10.21037/acs-2020-cfmcs-23
60. Mezzani A, Pistono $M$, Corrà U, et al. Systemic perfusion at peak incremental exercise in left ventricular assist device recipients: Partitioning pump and native left ventricle relative contribution. IJC Heart \& Vessels 2014;4:40-5. 